\title{
Analysis and Classification of Dermoscopic Images Using Spectral Graph Wavelet Transform
}

\author{
Kotra Sankar Rajasekhar ${ }^{1 *}$, Tummala Ranga Babu² \\ 1 Department of Electronics and Communication Engineering, Acharya Nagarjuna University, 522508 Guntur, Andhra Pradesh, India \\ 2 Department of Electronics and Communication Engineering, Rayapati Venkata Ranga Rao and Jagarlamudi Chandramouli \\ College of Engineering and Technology, 522019 Guntur, Andhra Pradesh, India \\ * Corresponding author, e-mail: rajsekharkotra@gmail.com
}

Received: 01 October 2019, Accepted: 23 December 2019, Published online: 25 March 2020

\begin{abstract}
The abnormal growth of skin cells leads to skin cancer which occurs due to the unrepaired DNA impairment to the skin cells. Worldwide every year more than 1.23 lakhs of skin cancers are diagnosed, out of which Melanoma is the deadliest one. The aim of this research work is Recognition of Melanoma and skin lesion classification from the Dermoscopic images. Feature extraction of Dermoscopic images can be done by Shape, Color and Texture features. Texture features comprises of Gray Level Co-occurrence Matrix (GLCM) and statistical texture features calculated from the coefficients of the Multiresolution transforms such as Discrete Wavelet Transform (DWT), Curvelet, Tetrolet, and Spectral Graph Wavelet Transform (SGWT). The novelty in this work is using SGWT for extraction of texture features. The superiority of SGWT over conventional wavelet transform is its ability to work on irregular shaped images. Weighted graphs are the base for the SGWT which can be obtained in the form of meshes for irregular shapes. In the present work, skin lesions are obtained from the International Skin Imaging Collaboration (ISIC) 2016 archive. The features obtained from the Dermoscopic images are classified using Naïve Baye's, K-Nearest Neighbor (KNN) and Support Vector Machine (SVM) classifiers. The proposed method using Shape, Color and Texture based features for Melanoma Recognition with SGWT results in an Area Under the Curve (AUC) of 0.951 with Accuracy of $96.79 \%$, Sensitivity of $88 \%$ and Specificity of $98.26 \%$. Further, the AUC of skin lesion classification such as Melanoma vs Nevus, Seborrheic Keratosis vs Squamous Cell Carcinoma, Melanoma vs Seborrheic Keratosis, Melanoma vs Basal Cell Carcinoma and Nevus vs Basal Cell Carcinoma using SGWT is $0.895,0.945,0.9645,0.945$ and 0.98 respectively.
\end{abstract}

\section{Keywords}

Dermoscopic images, SGWT, Melanoma, Nevus, Seborrheic Keratosis, Squamous Cell Carcinoma, Basal Cell Carcinoma, Naive Bayes, KNN, SVM classifier

\section{Introduction}

Skin Cancer is the leading cancer in the present era due to excessive exposure to sun, precancerous skin lesions, poor immunity, etc. Skin cancer are of various types like Actinic Keratosis, Basal Cell Carcinoma (BCC), Melanoma, Nevus, Squamous Cell Carcinoma (SCC), Seborrheic Keratosis (SK). Melanoma originates from the pigment-producing melanocytes that occurs rarely but is more dangerous. Every year more than 10000 people are dying due to Melanoma cancer [1]. Even though the naked eye can detect the skin lesions, starting stage Melanomas are hard to differentiate from benign with identical appearance leading to missed Melanomas although repeated skin biopsies are done.
Nowadays automated systems are playing an important role in diagnosing and evaluating the medical images especially, skin lesions. Computer aided diagnostic system is the main source to detect the skin cancer in the initial stages which leads to early treatment process. The input for any CAD system is an image which can be acquired using different medical imaging techniques.

The clinical examination of the skin lesion using naked eye assess the morphological features such as size, shape, color, contour and surface topology. This information is obtained only from the superficial layer of the skin. Hence, different non-invasive methods are to be used to acquire the skin lesions. The non-invasive method of 
diagnosing skin cancer involves acquisition of skin lesions using Microscopy, Electrical Impedance Spectroscopy (EIS), Optical Coherence Tomography (OCT) and Dermoscopy. Microscopic images are acquired using standard cameras or mobile phones whereas Dermoscopic images are acquired using a magnificent device with gel/oil interface or utilizing cross polarized light filters.

The standard process to acquire the data from skin is using Dermoscope which is a hand held device that contains a light source and a magnification lens. It is classified into Non Polarized Dermoscopy (NPD) and Polarized Dermoscopy (PD). Non Polarized Dermoscopy requires the glass plate to be in the direct contact with the skin surface using different kinds of immersion liquids like alcohol gel, water or mineral oil. NPD can visualize only the structures located in the epidermis and dermal-epidermal junction, but it fails to visualize the structures deeper than the dermal-epidermal junction.

Polarized Dermoscopy uses two polarized filters to attain cross polarization to avoid the direct contact with the skin and there is no need to use of immersion liquids. Polarized Dermoscopy allows better visualization structure of deeper skin layers. Dermoscopy images are used for the present work because visualization of additional color and pattern properties of the skin lesions can be obtained which increases the diagnosis accuracy [2].

\section{Related works}

Albahar [3] have proposed a convolution neural network with novel regulizer for skin lesion classification achieving an AUC of $0.77,0.93,0.85$, and 0.86 for Melanoma versus Nevus, Basal Cell Carcinoma versus Seborrheic Keratosis, Melanoma versus Seborrheic Keratosis, Melanoma versus Solar Lentigo respectively.

Zhang et al. [4], have proposed automatic skin lesion classification with skin lesions from ISIC skin 2017 dataset using attention residual learning Convolution Neural Network model achieving an AUC of 91.7. The trained model has taken only 0.2 seconds to classify a test image.

Mahbod et al. [5], proposed a fully automated system for skin lesion classification which involves an ensemble method for Convolution Neural Network (CNN) yielding an AUC of $87.3 \%$.

Barata et al. [6] have reviewed the different blocks in a CAD system. The most important block being the feature extraction that plays an important role in skin lesion classification. Feature extraction can be done by hand crafted, dictionary based, deep learning and clinically inspired features. Hand crafted features are shape, color and visual texture features.

$\mathrm{Yu}$ et al. [7], proposed hybrid classification of Dermoscopic images using Deep Convolution Neural Network, Fisher vector and SVM achieving an AUC of $79.57 \%$.

Codella et al. [8], proposed Melanoma Recognition in Dermoscopic images by combining hand crafted features such as color histogram, edge histogram, MSLBP, dictionary based features obtained using sparse coding and $\mathrm{CNN}$ achieving an AUC of $83.8 \%$.

Oliveira et al. [9], performed diagnosis of skin lesions using features such as color variation, texture analysis and shape properties. The texture features are based on fractal dimension analysis, DWT and Co-occurrence matrix. The evaluation of the proposed features is done by using different classifiers. Out of these OPF achieved $92.3 \%$ maximum accuracy, $87.5 \%$ sensitivity and $97.1 \%$ specificity with entire set of features.

Papanastasiou [10] proposed deep learning model designed from scratch as well as the pertained models Inception v3 and VGG-16 are used for Melanoma detection achieving an accuracy of $91.4 \%$ and $86.3 \%$ respectively.

$\mathrm{Yu}$ et al. [11], proposed automated Melanoma recognition using a fully convolution residual network for skin lesion segmentation. The proposed FCRN is integrated with a very deep residual network that enables the classification achieving an AUC of 0.826, 0.801 and 0.783 using VGG-16, Google Net and DRN-50 networks.

Vasconcelos and Vasconcelos [12] investigated that a deep learning convolution neural network based classifier can be used to deal with small and unbalanced data from ISBI 2016 achieving an accuracy of $82.5 \%$.

In this work, Melanoma recognition and skin lesions classification is done using Dermoscopic images by shape, color and texture features [13]. Texture features are obtained from both GLCM and the coefficients of the Multiresolution transforms such as Curvelet [14], Tetrolet [15], DWT [16] and SGWT [17]. The extracted shape, color and texture features are classified using Naïve Bayes [18], KNN [19] and SVM [20] classifiers. Section 3 gives the proposed work in which an overview of SGWT is given along with the methodology. Section 4 presents the experimental results obtained for Melanoma recognition and skin lesion classification. Section 5 gives conclusions and forthcoming work. 


\section{Proposed work}

\subsection{Spectral Graph Wavelet Transform}

A classical wavelet can be constructed by transforming and increasing or decreasing the range of single mother wavelet. By using these transformed and scaled waveforms the transform coefficients will be accustomed by the inner products of the input functionality. Further extension of this type of approach to arbitrary weighted graph causes some problems because for an irregular graph scaling and translation cannot be defined clearly. One way of dealing this kind of problem is by employing a spectral graph domain, by considering the basis into account which consists of Eigen functions of the Laplacian graph.

The SGWT proposed by Hammond et al. [17] is generated by wavelet operators that are valued operator functions of the Laplacian. The low frequency content of a function can be encoded using a second class of waveforms in SGWT [21].

A weighted graph $G=\{E, V, w\}$ consists of set of edges $E$, vertices $V$ taking in to consideration only finite graphs $|V|=N<\infty$, and weight function $w: E \rightarrow R^{+}$that allots to each edge a positive weight. For a weighted graph $G$, the adjacency matrix $A$ is the $N \times N$ matrix with entries $a_{m, n}$ where

$a_{m, n}= \begin{cases}w(e) & \text { if } e \in E \text { connects vertices } m \text { and } n \\ 0 & \text { otherwise. }\end{cases}$

Every vertex has a degree $d(m)$ for a weighted graph given by summation of weights of all the edges incident to it given by $d(m)=\sum_{n} a_{m, n}$. The matrix $D$ is defined to have diagonal elements equal to the degrees, and zeros elsewhere. The vertices of the graph have functions that are defined by $f \in R^{N}$ and $f(m)$ for the values on the vertex. The non-normalized Laplacian is defined by the spectral graph domain as

$£=D-A$.

For any $f \in R^{N}, £$ satisfies

$$
(£ f)(m)=\sum_{m \sim n} a_{m, n} \cdot(f(m)-f(n)) \text {. }
$$

The summation upon $m \sim n$ denotes addition of every vertices $n$ which are joined to the vertex $m$, and $a_{m, n}$ represents the weight of the edge joining $m$ and $n$.

The Laplacian operator in one dimension forms the Eigen functions that are complex exponential in the Fourier Transform. The inverse Fourier transform is the extension of $f$ in terms of Eigen functions of the Laplacian operator given by

$f(x)=\frac{1}{2 \pi} \int \hat{f}(\omega) e^{i \omega x} d \omega$.
A complex set of orthonormal Eigen vectors are contained in the graph Laplacian $£$ that is a real symmetric matrix. The Eigen vectors given by $x_{l}$ for $1=0, \ldots \ldots, N-1$ with correlated Eigen values $\lambda_{l}$ which are real

$£_{\chi_{l}}=\lambda_{l \chi_{l}}$.

The Graph Fourier transform $\hat{f}$ for the vertices on $G$ for any function $f \in R^{N}$ is given by

$\hat{f}(l)=\chi_{l}, f=\sum_{n=1}^{N} \chi_{l}^{*}(n) f(n)$.

The inverse transform is given by

$f(n)=\sum_{l=0}^{N-1} \chi_{l}(n) \hat{f}(l)$.

The kernel function $g: R^{+} \rightarrow R^{+}$which behaves as band pass filter is chosen as base for the spectral graph wavelet and need to satisfy the specifications $g(0)=0$ and $\lim _{x \rightarrow \infty} g(x)=0$. For the spectral graph wavelet kernel $g$, the wavelet operator $T_{g}=g(£)$ acts on a given function $f$ by modulating each Fourier mode as

$\widehat{T_{g}} f(l)=g\left(\lambda_{l}\right) \hat{f}(l)$.

The Inverse Fourier transform is given by

$\left(T_{g} f\right)(m)=\sum_{l=0}^{N-1} g\left(\lambda_{l}\right) \hat{f}(l) \chi_{l}(m)$.

The operator of the wavelet at scale $t$ can be indicated by $T_{g}^{t}=g(t L)$. Applying impulse on single vertex by localizing $T_{g}^{t}$ yields spectral graph wavelets

$\psi_{t, n}=T_{g}^{t} \delta_{n}$

The graph domain can be elaborated by

$\psi_{t, n}(m)=\sum_{l=0}^{N-1} g\left(t \lambda_{l}\right) \chi_{l}^{*}(n) \chi_{l}(m)$.

The wavelets $\psi_{t, n}(m)$ relies on the value of $g\left(t \lambda_{l}\right)$ only for $\lambda$ in the spectrum of $£$ which results in selection of scales require the knowledge of spectrum. For a function $f$, the wavelet coefficients are given by calculating the inner product with these wavelets, as

$W_{f}(t, n)=\psi_{t, n}, f$.

The wavelet operators are used to obtain wavelet coefficients directly using the orthogonality of $\left\{\chi_{l}\right\}$ by

$W_{f}(t, n)=\left(T_{g}^{t} f\right)(n)=\sum_{l=0}^{N-1} g\left(t \lambda_{l}\right) \hat{f}(l) \chi_{l}(n)$.

The low frequency of $f$ defined on the vertices can be efficiently represented by low pass residual scaling 
function which is determined by $h: R^{+} \rightarrow R^{+}$which acts as a low pass filter and satisfies the condition $h(0)>0$ and $h(x) \rightarrow 0$ as $x \rightarrow \infty$. The scaling functions are given by

$D_{n}=T_{h} \delta_{n}=h(L) \delta_{n}$.

The scaling coefficients are given by

$S_{f}(n)=D_{n}, f$.

Spectral graph wavelets can be localized in both frequency and time similar to the conventional wavelets by approximating $g(t L)$ by a lower order polynomial in $L$ as $t \rightarrow 0$ which is made possible using Taylor polynomial of $g$ at the origin.

\subsection{Methodology}

The present work aims at Recognition of Melanoma and classification of skin Lesions in Dermoscopic images. Skin lesions needs to be pre-processed to remove acquisition artifacts such as rulers, ink marks, reflections, air bubbles and cutaneous artifacts such as skin lines, blood vessels and thin lines by using a weighted average filter. Region growing technique is applied on the preprocessed skin lesions for acquiring the segmented images that are subjected to extraction of features such as Shape, Color and Texture (GLCM \& Multiresolution).

The various Multiresolution approaches used in this work are DWT, Curvelet, Tetrolet and SGWT. In this work SGWT plays an important role in extraction of features that improves the accuracy of Melanoma recognition and classifying skin lesions in Dermoscopic images. Classification can be done using different classifiers. The complete Methodology for this process is depicted in Fig. 1.

\subsubsection{Dataset}

The dataset for this work is taken from International Skin Imaging Collaboration (ISIC) archive ISBI challenge 2016 [22]. The data set provides Meta data such as clinical information, dimensions of the image, diagnosis, localization. The data set consists of 379 test images and 900 training images which are of different sizes ranging from $4288 \times 2848$ to $722 \times 542$ which are used for Melanoma recognition. Further the dataset also consists of different skin lesions such as 586 Basal Cell Carcinoma, 2169 Melanoma, 18566 Nevus, 419 Seborrheic Keratosis, and 226 Squamous Cell Carcinoma which are used for skin lesion classification. The skin lesions obtained from the dataset are shown in Fig. 2.

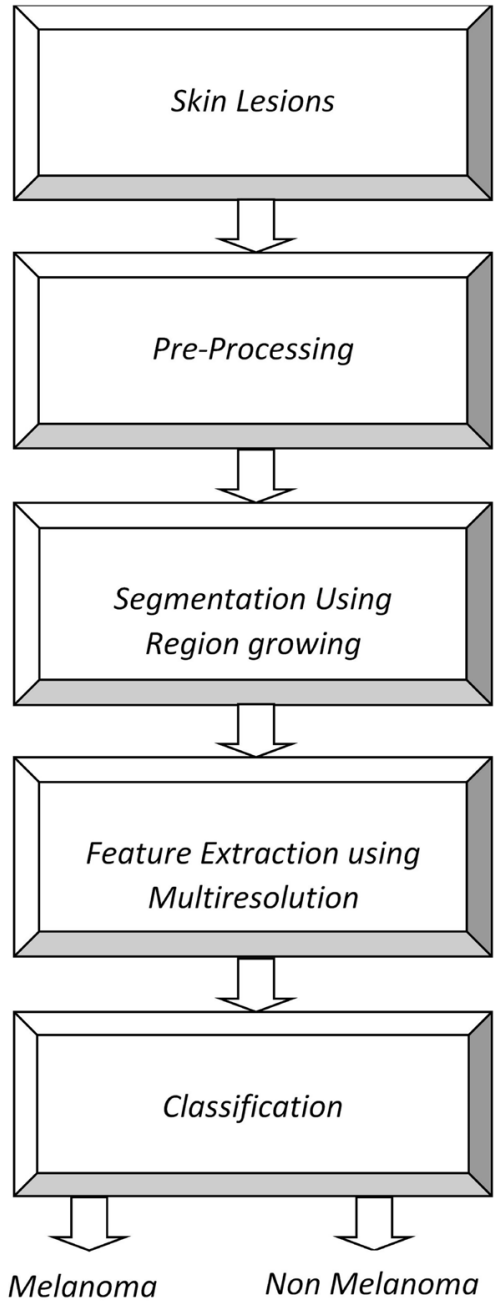

Fig. 1 Methodology for Melanoma recognition

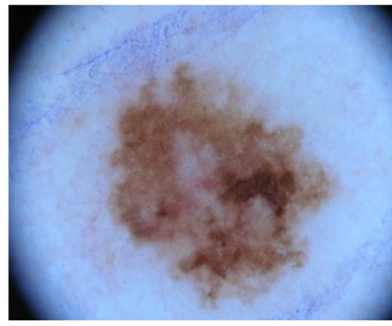

Melanoma_ISIC_0000002

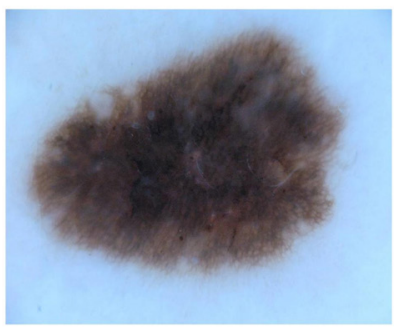

Nevus_ISIC_0000000

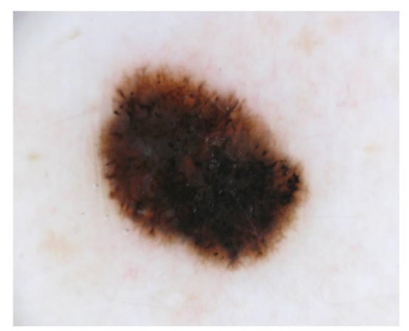

Melanoma_ISIC_0000013

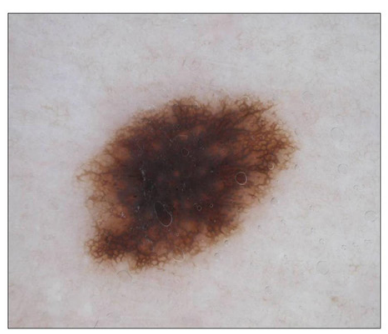

Nevus_ISIC_0000028
Fig. 2 ISIC Archive Data Set 2016 [22] 


\subsubsection{Segmentation}

The raw images are pre-processed and segmentation of the required tumor part is done by using region growing technique, in which a seed point is selected initially and from this point regions are grown by appending to the seed, those neighboring pixels that have properties similar to the seed. The segmented images are shown in the Fig. 3.

\subsubsection{Feature extraction}

Any characteristic of an image that helps to distinguish or discriminate an image from other images is called an image feature that is a function of quantified measurements and are required for image recognition. In this work, Shape, Color and Texture features are extracted from the different Dermoscopic images. The shape features include Area, Perimeter, Form Factor, Major Axis Length and Mass Deficient Coefficient (MDC) that are obtained from the shapes of skin lesion. Fig. 4 depicts the extraction of shape from skin lesions.

The values of Mean, Standard Deviation, Kurtosis and Skewness are calculated from each channel (RGB) of the color histogram and coefficients of the Multiresolution transform.

The texture features obtained from the GLCM such as Autocorrelation, Contrast, Correlation, Dissimilarity, Energy, Entropy, Homogeneity, Variance and Normalized Inverse Difference Moment are extracted for different skin lesion are shown in Table 1.

The Texture features calculated from the coefficients of different transforms for Melanoma, is show in Table 2. Shape features obtained for different skin lesions are shown in Table 3.

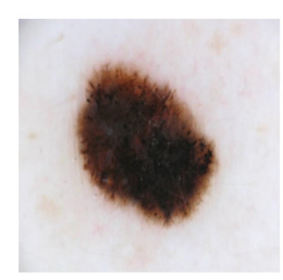

ISIC_0000013

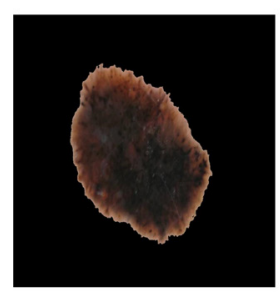

Segmented_ISIC_0000013

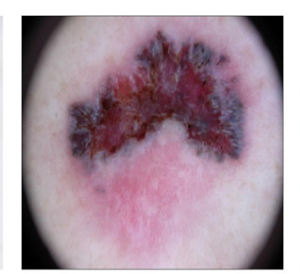

ISIC_0000030

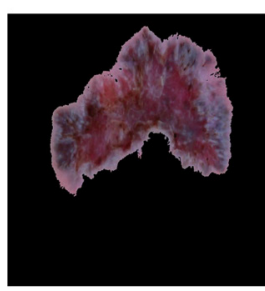

Segmented_ISIC_0000030

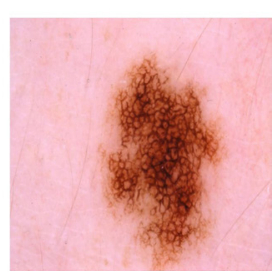

ISIC_0000197

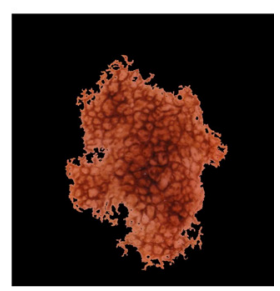

Segmented ISIC 0000197
Fig. 3 Segmentation of Dermoscopic images

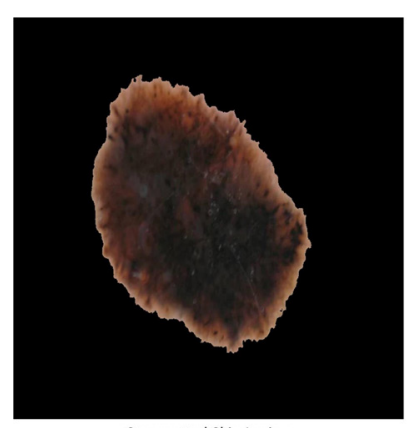

Segmented Skin Lesion

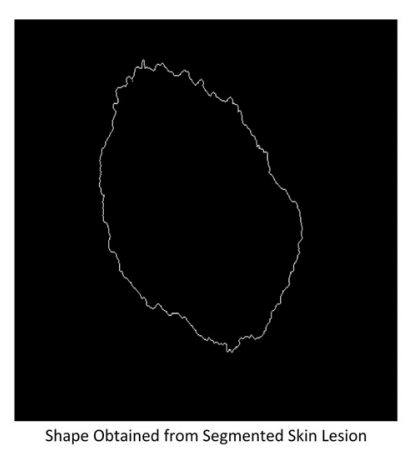

Shape Obtained from Segmented Skin Lesion
Fig. 4 Extraction of shape from segmented skin lesion

The texture features calculated from the coefficients of the Multiresolution transforms is a technique of sorting and processing the image in multiple resolutions using DWT, Curvelet, Tetrolet and SGWT.

The decomposition of a Dermoscopic image to get the wavelet coefficients at different scales by using SGWT is depicted in Fig. 5. The wavelet coefficients at scale 2 are considered in the present work since at other levels the Dermoscopic image losses most of the details.

\subsubsection{Classification}

Classification is a supervised learning method which involves training and testing phase. The classifier algorithm is feeded with a large set of known data in the training phase. The second phase is called testing phase where the constructed model is tested and evaluated with unknown test data. The classification task is descriptive as well as predictive. In this work Naive Bayes, KNN and SVM classifiers are used for classifying the features extracted from the Dermoscopic images.

The features obtained are used for Melanoma recognition and different skin lesion classification in the Dermoscopic images. Classification of skin lesion includes Melanoma vs Nevus, Melanoma vs Basal Cell Carcinoma, Melanoma vs Seborrheic Keratosis, Squamous Cell Carcinoma vs Seborrheic Keratosis and Nevus vs Basal Cell Carcinoma.

The feature vector comprising of Shape, Color and Texture features need to be standardized using Z-Score since the feature set has a mixture of positive and negative values. These features are given as an input to the classifier.

\section{Experimental results}

The classification accuracy using shape, color and texture features calculated from various transform techniques are shown in Table 4 which are evaluated using Naïve Bayes, KNN and SVM classifiers. The performance of 
Table 1 GLCM features obtained for different skin lesion

\begin{tabular}{lcccc}
\hline Feature & $\begin{array}{c}\text { Basal Cell Carcinoma } \\
\text { ISIC_0031442 }\end{array}$ & $\begin{array}{c}\text { Melanoma } \\
\text { ISIC_0000013 }\end{array}$ & $\begin{array}{c}\text { Nevus } \\
\text { ISIC_000003 }\end{array}$ & $\begin{array}{c}\text { Seborrheic Keratosis } \\
\text { ISIC_0001104 Squamous Cell Carcinoma } \\
\text { ISIC_0024418 }\end{array}$ \\
\hline Autocorrelation & 25.37605 & 21.85659 & 24.12864 & 25.3821 \\
Contrast & 0.38571 & 0.310915 & 0.369363 & 0.308004 \\
Correlation & 0.979844 & 0.984737 & 0.98056 & 0.985034 \\
Dissimilarity & 0.107989 & 0.078171 & 0.101754 & 0.075843 \\
Energy & 0.313244 & 0.399349 & 0.312298 & 0.354136 \\
Entropy & 1.554115 & 1.233506 & 1.597997 & 1.376708 \\
Homogeneity & 0.966896 & 0.976595 & 0.96762 & 0.057828 \\
Variance & 25.44414 & 21.90483 & 24.19329 & 0.978048 \\
Normalized Inverse & 0.995607 & 0.996746 & 0.99601 & 25.41433 \\
Difference Moment & & & 0.98527351 \\
\hline
\end{tabular}

Table 2 Texture features obtained for Melanoma skin lesions using DWT, Curvelet, Tetrolet and SGWT

\begin{tabular}{|c|c|c|c|c|c|c|}
\hline \multicolumn{7}{|c|}{ Melanoma } \\
\hline Skin Lesion & Transform & Mean & $\begin{array}{l}\text { Standard } \\
\text { Deviation }\end{array}$ & Smoothness Index & Skewness & Kurtosis \\
\hline \multirow{4}{*}{ ISIC_0000013 } & DWT & 0.713026 & 0.903313 & 0.449331 & 0.664454 & 1.528824 \\
\hline & CURVELET & 0.356411 & 0.453667 & 0.170685 & 0.663176 & 1.521723 \\
\hline & TETROLET & 1.426051 & 1.799227 & 0.763996 & 0.66345 & 1.531953 \\
\hline & SGWT & 0.25338 & 0.051218 & 0.002616 & 2.900938 & 11.49155 \\
\hline \multirow{4}{*}{ ISIC_0000145 } & DWT & 0.853843 & 0.899883 & 0.44745 & 0.367269 & 1.30928 \\
\hline & CURVELET & 0.426959 & 0.451762 & 0.169497 & 0.369623 & 1.305742 \\
\hline & TETROLET & 1.707686 & 1.792924 & 0.762728 & 0.375074 & 1.318716 \\
\hline & SGWT & 0.025782 & 0.049738 & 0.002468 & 2.969308 & 12.07572 \\
\hline
\end{tabular}

Table 3 Shape features obtained for different skin lesions

\begin{tabular}{lcccc}
\hline & Basal Cell Carcinoma & Melanoma & Nevus & \multicolumn{2}{c}{$\begin{array}{c}\text { Seborrheic Keratosis } \\
\text { Shape Feature }\end{array}$} & ISIC_0031442 & ISIC_0000013 & ISIC_0000003 & ISIC_0001104 \\
& 147203 & 123891 & 141693 & 139295 \\
Area & 7445 & 6152 & 6802 & 7549 \\
Perimeter & 0.0334 & 0.0411 & 0.0385 & 0.0307 \\
Form Factor & 610.8980 & 636.3517 & 602.2949 & 6.0418 \\
Major Axis Length & 583.0283 & 607.9734 & 598.2974 & 718.5958 \\
Minor Axis Length & & & 680.4115 & 590.4244 \\
\hline
\end{tabular}

the classification is evaluated using Accuracy, Sensitivity and Specificity that are based on the outcomes of the classifiers according to predicted class and known class. The training and testing skin lesions are divided in the ratio of 80-20. The Naive Bayes classifier greatly simplify learning by assuming that features are independent of given class. This classifier is best suited for two class data and competes with the most sophisticated classifiers. The classification accuracy using SGWT is $90.1163 \%$ with Naïve Bayes Classifier.

SVM classifies the data by a hyperplane, which divides all the data points of one class from the other class. Different kernels of SVM are used for Melanoma recognition from
Dermoscopic images. The accuracy obtained using linear kernel of SVM with features of SGWT is $95.9535 \%$ which is highest compared with the other kernels of the SVM.

The polynomial and RBF kernel of the SVM are also used for classification which results in lower accuracy compared to linear and quadratic kernel. The KNN classifier is a non-parametric method used for classification which makes a clear distinction between the different features given as input achieving maximum accuracy of $96.7912 \%$ with SGWT features.

The sensitivity and specificity values calculated from the confusion matrix for different transform techniques are as shown in Table 5. The superiority of 

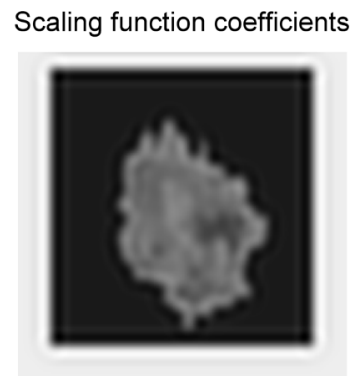

Wavelet coefficients at scale 3

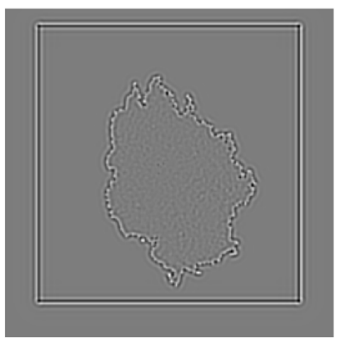

Wavelet coefficients at scale 1

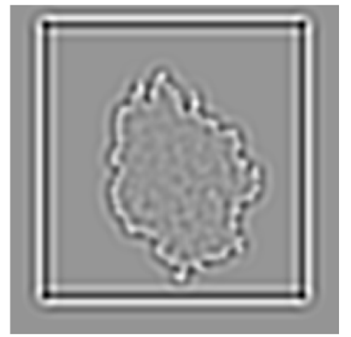

Wavelet coefficients at scale 4

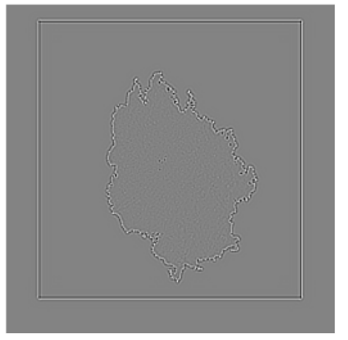

Wavelet coefficients at scale 2

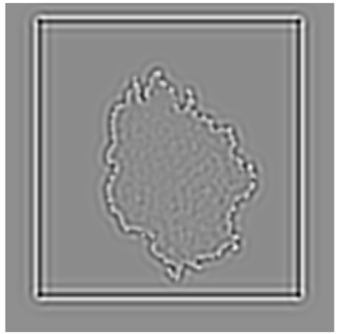

Wavelet coefficients at scale 5

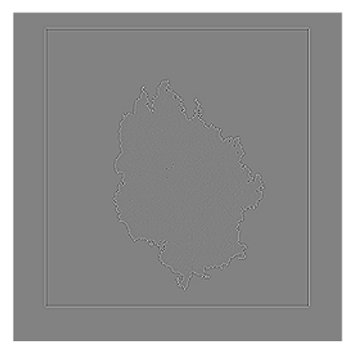

Fig. 5 Decomposition of Dermoscopic image ISIC_0000002 using SGWT

Table 4 Accuracy obtained for Melanoma recognition using different classifiers

\begin{tabular}{lccccc}
\hline \multirow{2}{*}{ Transform } & & \multicolumn{3}{c}{ Accuracy } \\
& Naive Bayes & SVM_Linear & SVM_RBF & SVM_Poly & SVM_Quad \\
\hline DWT & $88.116 \%$ & $94.4186 \%$ & $88.3721 \%$ & $90.1628 \%$ & $93.023 \%$ \\
CURVELET & $84.1628 \%$ & $94.8837 \%$ & $88.3721 \%$ & $91.023 \%$ & $93.5176 \%$ \\
TETROLET & $89.534 \%$ & $94.8837 \%$ & $88.6734 \%$ & $89.3023 \%$ & $93.488 \%$ \\
SGWT & $90.1163 \%$ & $95.9535 \%$ & $88.8372 \%$ & $92.8851 \%$ & $94.093 \%$ \\
\hline
\end{tabular}

the SGWT is due to the connectivity data which is embedded in the edge weights of the skin lesions and does not rely on any other features of vertices of the skin lesions.

Table 6 emphasizes the importance of different features in achieving the classification accuracy. The accuracy obtained using the combination of different features depicts that the use of shape, color and texture features results in better accuracy compared to combination of any other features. The texture features include features calculated from GLCM and coefficients of SGWT that plays a significant role in obtaining better accuracy.

The graph of Area Under the Curve (AUC) using Multiresolution transforms for Melanoma Recognition is

Table 5 Sensitivity and specificity values obtained by DWT, Curvelet, Tetrolet and SGWT using KNN classifier for Melanoma recognition

\begin{tabular}{lcc}
\hline Transform & Sensitivity (\%) & Specificity (\%) \\
\hline DWT & 85 & 95 \\
Curvelet & 86 & 96 \\
Tetrolet & 86 & 97 \\
SGWT & 88 & 98.26 \\
\hline
\end{tabular}

shown in Fig. 6. The AUC using DWT is less since it can resolve one dimensional singularity in horizontal and vertical directions only. The objects that exhibit curve-punctuated smoothness are represented by Curvelet transform. Hence, a Curvelet cannot capture the local variations in the skin lesions as a result AUC is less compared to Tetrolet and SGWT. The AUC using Tetrolet is less compared to SGWT since it fails to extract the information at the edges of the skin lesions because of the basic shapes of Tetriminoes. The AUC using SGWT is highest compared to the other transforms.

In this work skin lesion classification can also be done with Multiresolution transforms using KNN classifier since its performance is superior to that of Naïve Bayes and SVM

Table 6 Classification performance using different features for Melanoma recognition from Dermoscopic images.

\begin{tabular}{lc}
\hline Transform & Accuracy $(\%)$ \\
\hline Shape + Color & 92.2621 \\
Texture + Color & 94.231 \\
Shape + Texture & 95.1163 \\
Texture + Color + Shape & 96.7912 \\
\hline
\end{tabular}




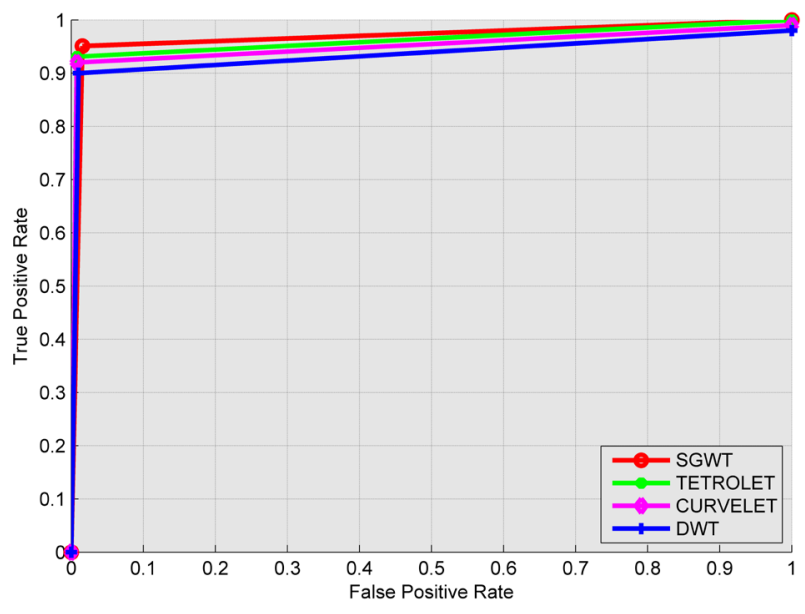

Fig. 6 AUC of Melanoma recognition using multiresolution transforms

for two class data. The classification of Melanoma vs Nevus, Seborrheic Keratosis (SK) vs Squamous Cell Carcinoma (SCC), Melanoma vs Seborrheic Keratosis (SK), Melanoma vs Basal Cell Carcinoma (BCC) and Nevus vs Basal Cell Carcinoma (BCC) is done to distinguish Melanoma from other types of skin lesions. The features extracted from these different lesions are given as input to KNN classifier. The AUC for classifying different skin lesions using different Multiresolution transforms is shown in Table 7 and the accuracy graph for different skin lesions is shown in Fig. 7 which depicts that SGWT has outperformed other transforms. Also the Sensitivity and Specificity values for classifying skin lesions using different Multiresolution transforms along with their values are shown in Figs. 8 and 9.

The sensitivity of Curvelet is highest 0.8482 compared to the SGWT, but the specificity value of SGWT dominates that of Curvelet along with the accuracy which is $89.5 \%$. The variations in the values of specificity, sensitivity and accuracy achieved using different transforms is less, depicting that Multiresolution transforms can resolve singularities along edges and boundaries of irregular shaped skin lesions.

The classification of Seborrheic Keratosis (SK) vs Squamous Cell Carcinoma (SCC) is the need of the hour as SCC arises within Seborrheic Keratosis. Different transforms are used to distinguish between the two skin lesions with Tetrolet transform having higher Sensitivity than SGWT which achieved better specificity and Accuracy rather than the other transforms.

Melanoma and Seborrheic Keratosis skin lesions were classified using different transforms with SGWT resulting in high Specificity and Sensitivity values rather than the other transforms since the base function defined over the irregular topology of the skin lesion provides a good representation for the related shape from which features can be extracted.

The skin lesions Melanoma and Basal Cell Carcinoma are both malignant in which Melanoma is a malignant disease which grows aggressively and spreads to other parts of the body unlike the Basal Cell Carcinoma which doesn't spread to other parts of the body. Classification of these lesions using Tetrolet transform yields better sensitivity while SGWT performance is superior in terms of Specificity and Accuracy. Finally, the classification of Nevus from Basal Cell Carcinoma with SGWT yields an Accuracy of $98 \%$ which is higher compared to the accuracies obtained using other transforms. The AUC graphs for classifying different skin lesions is shown in Figs. 10 to 14.

Table 8 shows the comparison of the proposed work with the existing literature. The previous works are done using Convolution Neural Network with ISIC 2016 dataset. The computational time to calculate the texture features from a skin lesion using DWT, Curvelet, Tetrolet and SGWT is 0.322, 4.298, 4.028, 14.11 seconds respectively, evaluated using MATLAB R2017a Version No 9.2 on $2.30 \mathrm{GHz}$ Intel Core i5 processor. The superiority of the proposed work compared to the existing literature prove that Multiresolution transform resolve the singularities present in the irregular shaped skin lesions.

\section{Conclusion}

In this work, Melanoma recognition from Dermoscopic images and classification of skin lesions obtained from the ISIC archive 2016 is done. The features are extracted using both shape, color and texture features.

Table 7 AUC values for classifying skin lesions using different Multiresolution transforms

\begin{tabular}{|c|c|c|c|c|c|}
\hline \multirow[b]{2}{*}{ Transform } & \multicolumn{5}{|c|}{ AUC } \\
\hline & Melanoma vs Nevus & $\begin{array}{l}\text { Seborrheic Keratosis vs } \\
\text { Squamous Cell Carcinoma }\end{array}$ & $\begin{array}{l}\text { Melanoma vs } \\
\text { Seborrheic Keratosis }\end{array}$ & $\begin{array}{l}\text { Melanoma vs Basal } \\
\text { Cell Carcinoma }\end{array}$ & $\begin{array}{l}\text { Nevus vs Basal cell } \\
\text { Carcinoma }\end{array}$ \\
\hline DWT & 0.89 & 0.94 & 0.941 & 0.925 & 0.95 \\
\hline Curvelet & 0.89 & 0.93 & 0.94 & 0.94 & 0.97 \\
\hline Tetrolet & 0.89 & 0.925 & 0.94 & 0.942 & 0.975 \\
\hline SGWT & 0.895 & 0.945 & 0.9645 & 0.945 & 0.98 \\
\hline
\end{tabular}




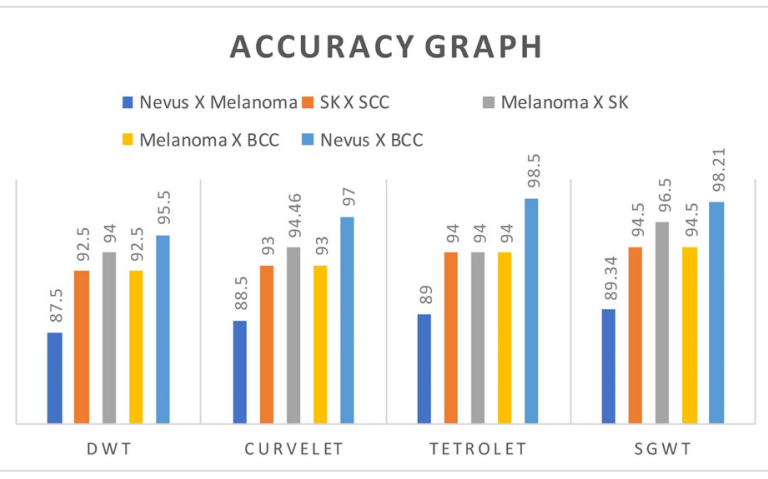

Fig. 7 Accuracy graph for classification of skin lesions using Multiresolution transforms

\section{SENSITIVITY GRAPH}

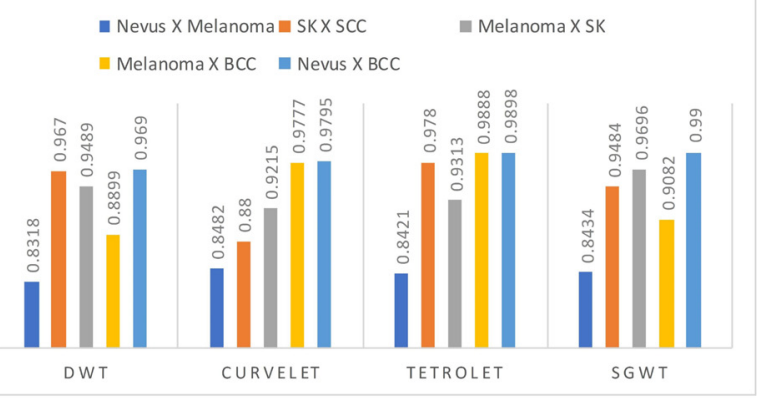

Fig. 8 Sensitivity graph for classification of skin lesions using Multiresolution transforms

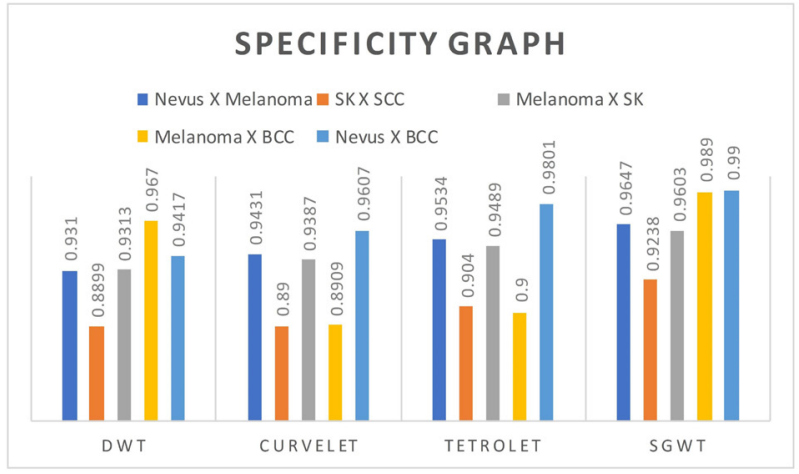

Fig. 9 Specificity graph for classification of skin lesions using Multiresolution transforms

The texture features are obtained from both GLCM and different Multiresolution transform. The most important part in this approach is SGWT that has the capability of allowing Multiresolution representation of shapes. In this work the results are evaluated using metrics such as AUC, sensitivity and specificity. The accuracy is increased by using the texture features calculated from the coefficients of SGWT rather than DWT, Curvelet and Tetrolet.

In the future work, Melanoma recognition and skin lesion classification can be done by combining Multiresolution

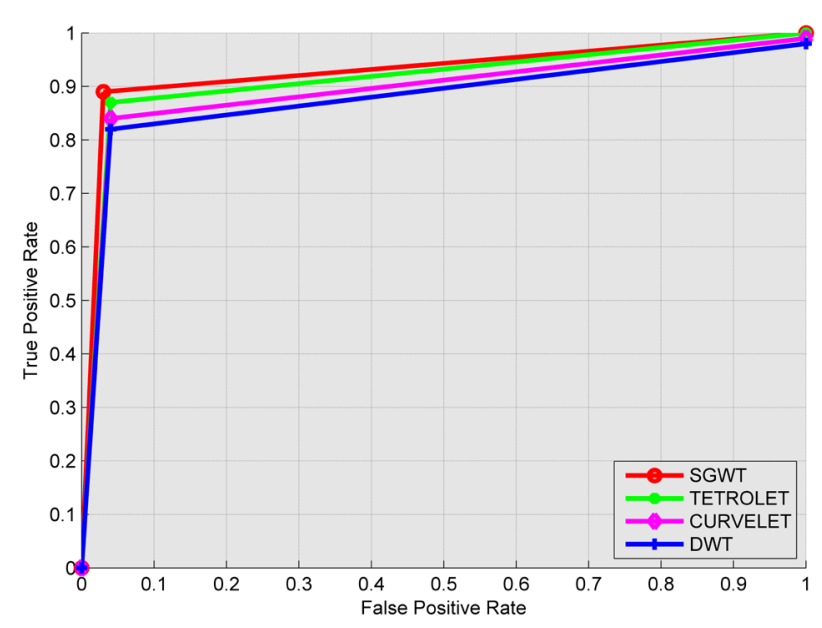

Fig. 10 AUC graph to classify Melanoma vs Nevus

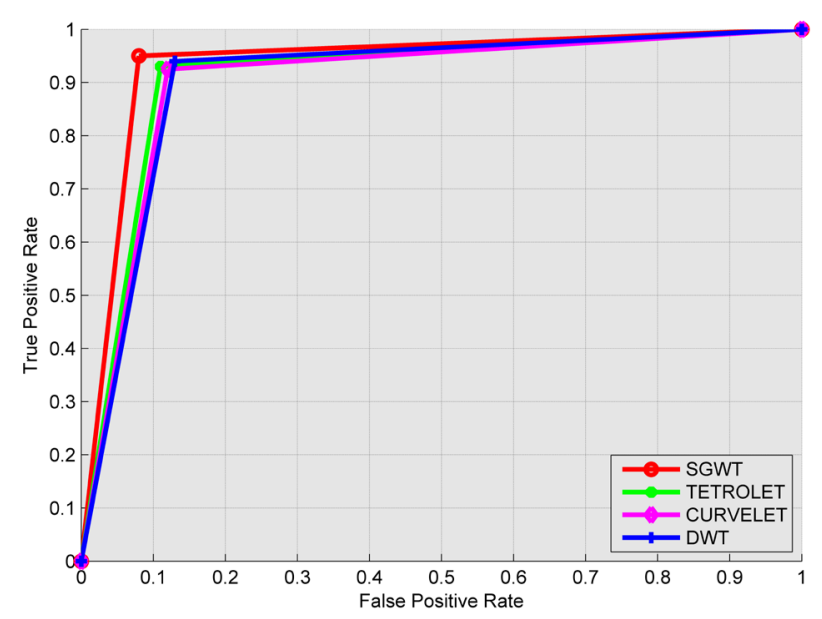

Fig. 11 AUC graph to classify Melanoma vs Seborrheic Keratosis

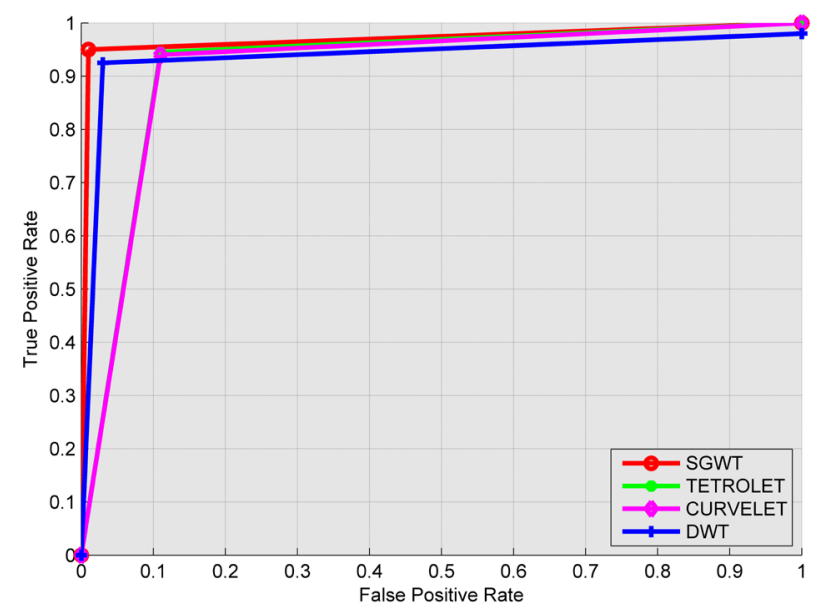

Fig. 12 AUC graph to classify Melanoma vs Basal Cell Carcinoma

transform with convolution neural networks which may improve the accuracy than the existing methods. 


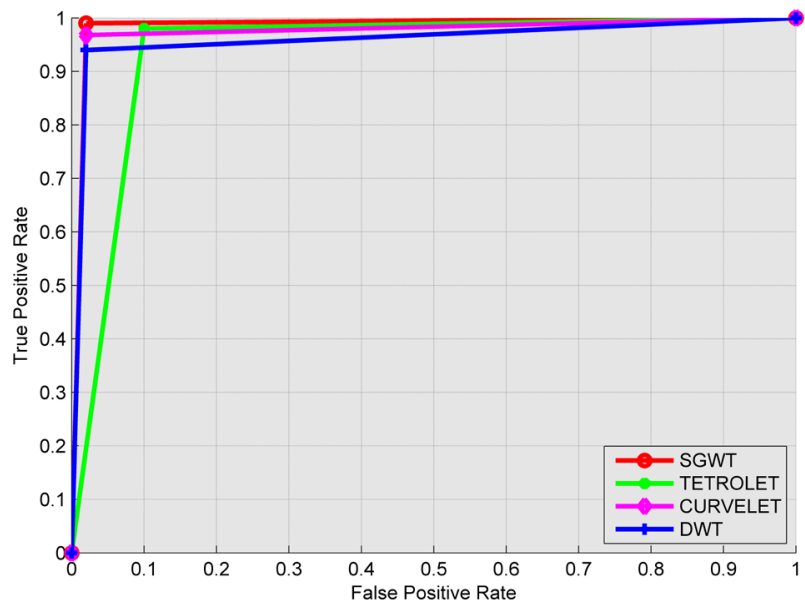

Fig. 13 AUC graph to classify Nevus vs Basal Cell Carcinoma

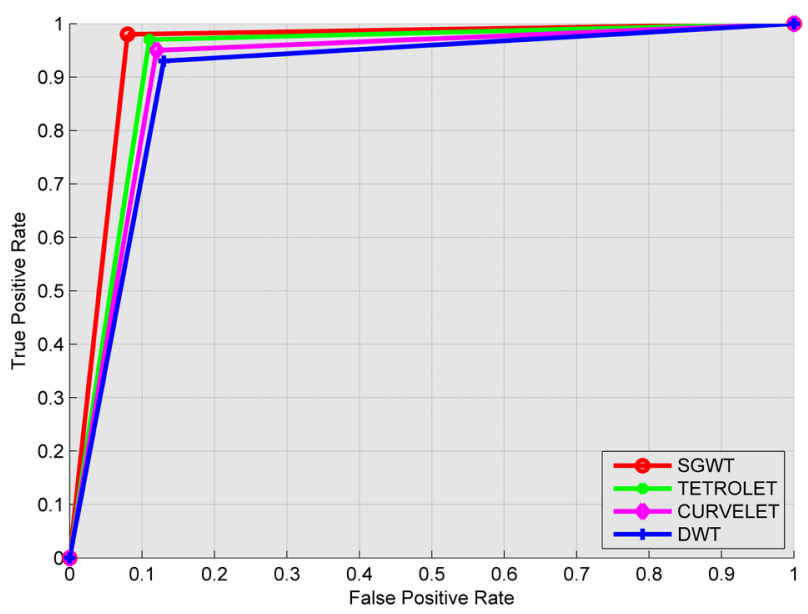

Fig. 14 AUC graph to classify Seborrheic Keratosis vs Squamous Cell Carcinoma

Table 8 Comparison of existing literature with the proposed work for Melanoma recognition

\begin{tabular}{|c|c|c|c|c|c|}
\hline Author & Method & Sensitivity $(\%)$ & Specificity (\%) & Accuracy $(\%)$ & AUC \\
\hline Demyanov et al. [23] & $\mathrm{CNN}$ with eight layers & - & - & $88 \%$ & - \\
\hline Yu et al. [11] & FCRN & 50.7 & 94.1 & 85.5 & 0.804 \\
\hline Yoshida et al. [24] & CNN classifier and Cytological findings & 80.9 & 88.1 & - & 0.84 \\
\hline Codella et al. [8] & Handcrafted,Dictionary, Deep Learning & 69.3 & 83.6 & 80.7 & 78.3 \\
\hline $\begin{array}{l}\text { Vasconcelos and } \\
\text { Vasconcelos [12] }\end{array}$ & Deep Learning (GoogleNet) & 74.6 & 84.5 & 82.5 & - \\
\hline Proposed work & SGWT with KNN classifier & 88 & 98.26 & 96.79 & 0.951 \\
\hline
\end{tabular}

\section{References}

[1] Chang, H. "Skin cancer reorganization and classification with deep neural network", [cs.CV] Computer Science: Computer Vision and Pattern Recognition, arXiv:1703.00534, Cornell University, Ithaca, NY, USA, 2017. [online] Available at: https://arxiv.org/ abs/1703.00534 [Accessed: 29 September 2019]

[2] Dermoscopedia "Principles of dermoscopy", [online] Available at: https://dermoscopedia.org/Principles_of_dermoscopy [Accessed: 29 September 2019]

[3] Albahar, M. A. "Skin Lesion Classification Using Convolutional Neural Network With Novel Regularizer", IEEE Access, 7, pp. 38306-38313, 2019.

https://doi.org/10.1109/ACCESS.2019.2906241

[4] Zhang, J., Xie, Y., Xia, Y., Shen, C. "Attention Residual Learning for Skin Lesion Classification", IEEE Transactions on Medical Imaging, 38(9), pp. 2092-2103, 2019.

https://oi.org/10.1109/TMI.2019.2893944

[5] Mahbod, A., Schaefer, G., Ellinger, I., Ecker, R., Pitiot, A., Wang, C. "Fusing fine-tuned deep features for skin lesion classification", Computerized Medical Imaging and Graphics, 71, pp. 19-29, 2019. https://doi.org/10.1016/j.compmedimag.2018.10.007

[6] Barata, C., Celebi, M. E., Marques, J. S. "A Survey of Feature Extraction in Dermoscopy Image Analysis of Skin Cancer", IEEE Journal of Biomedical and Health Informatics, 23(3), pp. 1096-1109, 2019.

https://doi.org/10.1109/JBHI.2018.2845939
[7] Yu, Z., Ni, D., Chen, S., Qin, J., Li, S., Wang, T., Lei, B. "Hybrid dermoscopy image classification framework based on deep convolutional neural network and Fisher vector", In: 2017 IEEE 14th International Symposium on Biomedical Imaging (ISBI 2017), Melbourne, Australia, 2017, pp. 301-304.

https://doi.org/10.1109/isbi.2017.7950524

[8] Codella, N., Nguyen, Q. B., Pankanti, S., Gutman, D., Helba, B., Halpern, A., Smith, J. R. "Deep Learning Ensembles for Melanoma Recognition in Dermoscopy Images", IBM Journal of Research and Development, 61(4-5), pp. 1-28, 2017. [online] Available at: https:// arxiv.org/abs/1610.04662 [Accessed: 29 September 2019]

[9] Oliveira, R. B., Pereira, A. S., Tavares, J. M. R. S. "Computational diagnosis of skin lesions from dermoscopic images using combined features", Neural Computing and Applications, 31(10), pp. 6091-6111, 2019.

https://doi.org/10.1007/s00521-018-3439-8

[10] Papanastasiou, M. "Use of Deep Learning in Detection of Skin Cancer and Prevention of Melanoma", MSc Thesis, KTH Royal Institute of Technology School of Technology and Health, 2017. [online] Available at: https://pdfs.semanticscholar.org/4ab5/8d71e8dd01fc0107b5b7cbc7179ff01e4d29.pdf [Accessed: 29 September 2019]

[11] Yu, L., Chen, H., Dou, Q., Qin, J., Heng, P.-A. "Automated Melanoma Recognition in Dermoscopy Images via Very Deep Residual Networks", IEEE Transactions on Medical Imaging, 36(4), pp. 994-1004, 2017.

https://doi.org/10.1109/TMI.2016.2642839 
[12] Vasconcelos, C. N., Vasconcelos, B. N. (in press) "Experiments using deep learning for dermoscopy image analysis", Pattern Recognition Letters, 2017.

https://doi.org/10.1016/j.patrec.2017.11.005

[13] Clausi, D. A. "An analysis of co-occurrence texture statistics as a function of grey level quantization", Canadian Journal of Remote Sensing, 28(1), pp. 45-62, 2002. https://doi.org/10.5589/m02-004

[14] Candès, E., Demanet, L., Donoho, D., Ying, L. "Fast Discrete Curvelet Transforms", Multiscale Modeling and Simulation, 5(3), pp. 861-899, 2006. https://doi.org/10.1137/05064182X

[15] Krommweh, J. "Tetrolet transform: A new adaptive Haar wavelet algorithm for sparse image representation", Journal of Visual Communication and Image Representation, 21(4), pp. 364-374, 2010. https://doi.org/10.1016/j.jvcir.2010.02.011

[16] Mallat, S. "A Wavelet Tour of Signal Processing", Academic Press, Burlington, MA, USA, 2009.

https://doi.org/10.1016/B978-0-12-374370-1.X0001-8

[17] Hammond, D. K., Vandergheynst, P., Gribonval, R. "Wavelets on graphs via spectral graph theory", Applied and Computational Harmonic Analysis, 30(2), pp. 129-150, 2011. https://doi.org/10.1016/j.acha.2010.04.005

[18] Murphy, K. P. "Naive Bayes classifiers", Institute of Computing, University of Campinas, Campinas, Brazil, 2006, Available at: https://www.ic.unicamp.br/ rocha/teaching/2011s1/mc906/aulas/ naive-bayes.pdf [Accessed: 29 September 2019]

[19] Dudani, S. A. "The Distance-Weighted k-Nearest-Neighbor Rule", IEEE Transactions on Systems, Man, and Cybernetics, SMC-6(4), pp. 325-327, 1976.

https://doi.org/10.1109/TSMC.1976.5408784
[20] Suykens, J. A. K., Vandewalle, J. "Least Squares Support Vector Machine Classifiers", Neural Processing Letters, 9(3), pp. 293300, 1999. https://doi.org/10.1023/A:1018628609742

[21] Masoumi, M., Hamza, A. B. "Shape classification using spectral graph wavelets", Applied Intelligence, 47(4), pp. 1256-1269, 2017. https://doi.org/10.1007/s10489-017-0955-7

[22] Gutman, D., Codella, N. C. F., Celebi, E., Helba, B., Marchetti, M., Mishra, N., Halpern, A. "Skin Lesion Analysis Toward Melanoma Detection: A Challenge at the International Symposium on Biomedical Imaging (ISBI) 2016, hosted by the International Skin Imaging Collaboration (ISIC)", [cs.CV] Computer Science: Computer Vision and Pattern Recognation, arXiv:1605.01397, Cornell University, Ithaca, NY, USA, 2016. [online] Available at: https://arxiv.org/pdf/1605.01397 [Accessed: 29 September 2019]

[23] Demyanov, S., Chakravorty, R., Abedini, M., Halpern, A., Garnavi, R. "Classification of dermoscopy patterns using deep convolutional neural networks", In: 2016 IEEE 13th International Symposium on Biomedical Imaging (ISBI), Prague, Czech Republic, 2016, pp. 364-368. https://doi.org/10.1109/ISBI.2016.7493284

[24] Yoshida, T., Celebi, M. E., Schaefer, G., Iyatomi, H. "Simple and Effective Pre-processing for Automated Melanoma Discrimination based on Cytological Findings", In: 2016 IEEE International Conference on Big Data (Big Data), Washington, DC, USA, 2016, pp. 3439-3442.

https://doi.org/10.1109/BigData.2016.7841005 